\title{
PATTERN OF STRESS-STRAIN ACCUMULATION DUE TO A LONG DIP- SLIP FAULT MOVEMENT IN A VISCOELASTIC LAYERED MODEL OF THE LITHOSPHERE -ASTHENOSPHERE SYSTEM
}

\author{
S.K. DEBNATH ${ }^{*}$ \\ Department of Basic Science and Humanities \\ Meghnad Saha Institute of Technology (A unit of Techno India Group) \\ Nazirabad P.O., Uchhepota \\ Via- Sonarpur, Kolkata-700150, INDIA \\ E-mail: skd.sxccal@gmail.com \\ S. SEN \\ Department of Applied Mathematics \\ University of Calcutta \\ 92, APC Road, Kolkata 700009, INDIA \\ E-mail: dr.sanjaysen@rediff.com
}

\begin{abstract}
The process of stress accumulation near earthquake faults during the aseismic period in between two major seismic events in seismically active regions has become a subject of research during the last few decades. In the present paper a long dip -slip fault is taken to be situated in a viscoelastic layer over a viscoelastic half space representing the lithosphere-asthenosphere system. A movement of the dip-slip nature across the fault occurs when the accumulated stress due to various tectonic reasons, e.g., mantle convection etc., exceeds the local friction and cohesive forces across the fault. The movement is assumed to be slipping in nature, expressions for displacements, stresses and strains are obtained by solving the associated boundary value problem with the help of integral transformation and Green's function method. A detailed study of these expressions may give some ideas about the nature of stress accumulation in the system, which in turn will be helpful in formulating an earthquake prediction programme.
\end{abstract}

Key words: aseismic period, dip-slip fault, earthquake prediction, stress accumulation, viscoelastic-layered model.

\section{Introduction}

It is the observational fact that while some faults are strike slip (finite or long ) in nature, there are faults (e.g., Sierra Nevada/Owens valley: Basin and Range faults, Rocky Mountains, Himalayas, Atalanti fault of central Greece- a steeply dipping fault with dip 60,80( $\mathrm{deg})$ ) where the surface level changes during the motion, i.e., the faults are dip-slip in nature.

It is therefore necessary to understand the mechanism of plate motion in the dip direction before the fault movement and after the fault movement with a displacement dislocation and to understand the nature of stress-strain accumulation/release in spacial and temporal co-ordinates to predict the future event in space and time.

A pioneering work involving static ground deformation in elastic media was initiated by Maruyama (1964; 1966). Savage and Hastie (1966) did a wonderful work in analyzing the displacement, stress and strain for dip-slip movement. Later some theoretical models in this direction have been formulated by a number of authors like Rybicki (1971; 1973), Sato (1972), Rosenman and Singh (1973; 1974), Nur and

\footnotetext{
* To whom correspondence should be addressed
} 
Mavko (1974), Sato and Yamashita (1975), Rundle (1978), Mukhopadhyay et al. (1979a; 1979b; 1980a; 1980b), Cohen (1980a), Sunita and Singh (1991; 1992), Ghosh et al. (1992), Singh et al. (1997), Savage (1998), Oglesby (2005), Zhang et al. (2006), Savage (2007), Ma and Beroza (2008). Segall (2010) discussed various aspects of fault movement in his book. Ghosh and Sen (2011) discussed stress accumulation near buried fault in the lithosphere-asthenosphere system. The study of Fuis et al. (2012) can also be mentioned.

In most of these works the media were taken to be elastic and /or viscoelastic, but a layered model with viscoelastic layer(s) over viscoelastic half space will be a more realistic one for the lithosphereasthenosphere system.

The following are some reasons behind our consideration of viscoelastic materials. The laboratory experiments on rocks at high temperature and pressure indicate the imperfect elastic behavior of the rocks situated in the lower lithosphere and asthenosphere.

Investigations on the post-glacial uplift of Fennoscandia and parts of Canada indicate that at the termination of the last ice age, which happened about 10 millennia ago a $3 \mathrm{~km}$ ice cover melted gradually leading and upliftment of the regions. Evidence of this upliftment has been discussed in the work of Fairbridge (1961), Schofield (1964), Chathles (1975). If the Earth were perfectly elastic, this deformation would be managed after the removal of the load, but it did not so happened, which indicates that the Earth crust and upper mantle is not perfect elastic but rather viscoelastic in nature. In the present case we consider a long dip-slip fault situated in a viscoelastic layer over a viscoelastic half space which reaches up to the free surface. The medium is under the influence of tectonic forces due to mantle convection or some related phenomena. The fault is assumed to undergo a slipping movement when the stresses in the region exceed certain threshold values.

In our paper, we consider a viscoelastic layer of thickness $H \mathrm{~km}$ (say) over a viscoelastic half space to represent the lithosphere-asthenosphere system, with constant rigidity $\left(2.0 \times 10^{5} \mathrm{Mpa}\right)$ and viscosity $\left(10^{20}\right.$ $-10^{21} \mathrm{pa} \cdot \mathrm{s}$ ) following the observational data mentioned by Chift et al. (2002). Shunichiro Karato (July, 2010). Analytical expressions for displacements, stresses and strains in the system are obtained both before and after the fault movement using are appropriate mathematical technique involving integral transformation and Green's function. Numerical computational work is carried out with suitable values of the model parameters and the nature of the stress and strain accumulation in the medium is investigated. It is expected that complete understanding of the stress-strain accumulation/release will be helpful in predicting the future event in space and time.

\section{Formulation}

We consider a long dip-slip fault $F$ and width $D$ situated in a viscoelastic layer over a viscoelastic half space of linear Maxwell type.

A Cartesian co-ordinate system is used with a suitable point $O$ on the strike of the fault as the origin, the $Y_{1}$ axis along the strike of the fault, the $Y_{2}$ axis is as shown in Fig. 1 and the $Y_{3}$ axis pointing downwards. We choose another co-ordinate system $Y_{1}^{\prime}, Y_{2}^{\prime}$ and $Y_{3}^{\prime}$ axes as shown in Fig.1 below, so that the fault is given by $F:\left(y_{2}^{\prime}=0,0 \leq y_{3}^{\prime} \leq D\right)$. Let $\theta$ be the dip of the fault $F$. Let $v_{k}, w_{k}$ be the displacement component along the $Y_{2}^{\prime}$ and $Y_{3}^{\prime}$ axes and $\tau_{i j}^{k}, e_{i j}^{k}, i, j=2,3$ be the stress and strain component associated with the dip-slip motion in the layer and half-space respectively for $k=1,2$. 


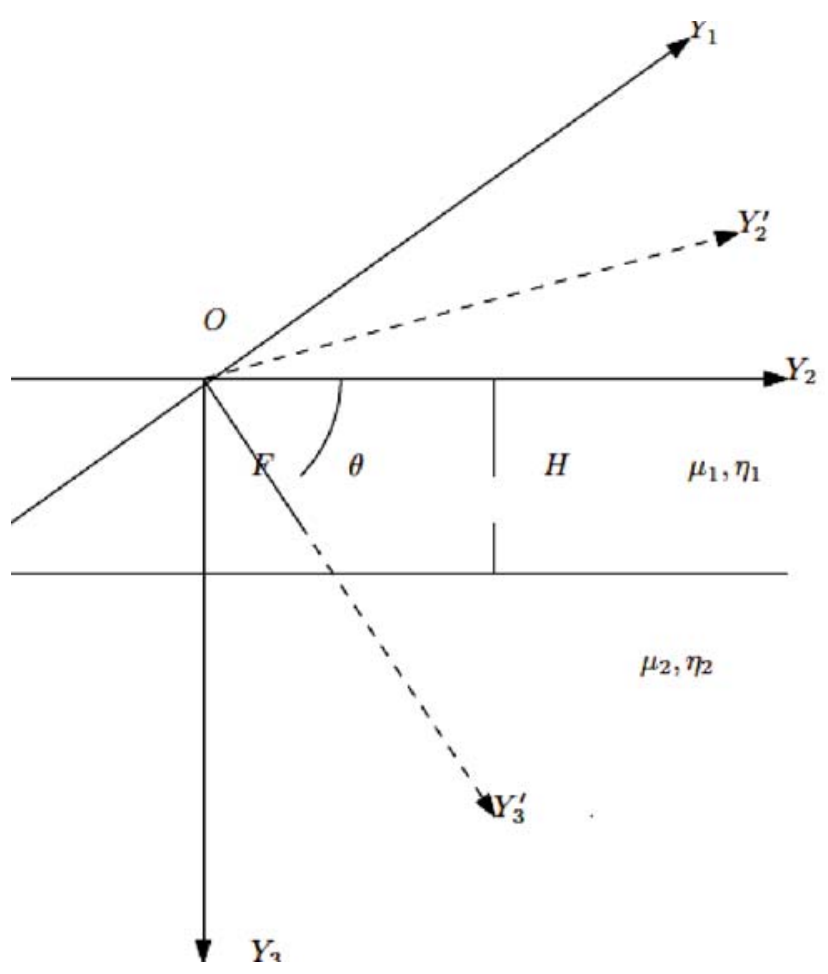

Fig.1. Section of the model by the plane $y_{1}=0$.

Then we have,

$$
Y=A Y^{\prime}
$$

where, $\quad Y=\left(\begin{array}{l}y_{1} \\ y_{2} \\ y_{3}\end{array}\right), \quad Y^{\prime}=\left(\begin{array}{l}y_{1}^{\prime} \\ y_{2}^{\prime} \\ y_{3}^{\prime}\end{array}\right) \quad$ and $\quad A=\left(\begin{array}{ccc}1 & 0 & 0 \\ 0 & \sin \theta & \cos \theta \\ 0 & -\cos \theta & \sin \theta\end{array}\right)$,

$\tau^{\prime}=B \tau$

where, $\quad \tau=\left(\begin{array}{c}\tau_{22} \\ \tau_{23} \\ \tau_{33}\end{array}\right), \quad \tau^{\prime}=\left(\begin{array}{c}\tau_{2^{\prime} 2^{\prime}} \\ \tau_{2^{\prime} 3^{\prime}} \\ \tau_{3^{\prime} 3^{\prime}}\end{array}\right) \quad$ and $\quad B=\left(\begin{array}{ccc}\sin ^{2} \theta & \sin 2 \theta & \cos ^{2} \theta \\ (-1 / 2) \sin 2 \theta & -\cos 2 \theta & (1 / 2) \sin 2 \theta \\ \cos ^{2} \theta & -\sin 2 \theta & \sin ^{2} \theta\end{array}\right)$.

\subsection{For a viscoelastic Maxwell type medium the constitutive equations are taken as}

For the layer: $M 1$ and for half-space: $M 2$

$$
\left(\frac{1}{\eta_{k}}+\frac{1}{\mu_{k}} \frac{\partial}{\partial t}\right) \tau_{22}^{k}=\frac{\partial}{\partial t}\left(e_{22}^{k}\right)=\frac{\partial}{\partial t}\left(\frac{\partial v_{k}}{\partial y_{2}}\right)
$$




$$
\begin{aligned}
& \left(\frac{1}{\eta_{k}}+\frac{1}{\mu_{k}} \frac{\partial}{\partial t}\right) \tau_{23}^{k}=\frac{\partial}{\partial t}\left(e_{23}^{k}\right)=\left(\frac{1}{2}\right) \frac{\partial}{\partial t}\left(\frac{\partial v_{k}}{\partial y_{3}}+\frac{\partial w_{k}}{\partial y_{2}}\right), \\
& \left(\frac{1}{\eta_{k}}+\frac{1}{\mu_{k}} \frac{\partial}{\partial t}\right) \tau_{33}^{k}=\frac{\partial}{\partial t}\left(e_{33}^{k}\right)=\frac{\partial}{\partial t}\left(\frac{\partial w_{k}}{\partial y_{3}}\right)
\end{aligned}
$$

where, $k=1$ for the layer and $k=2$ for the half-space.

where, $\mu_{1}, \mu_{2}$ are the effective rigidity and $\eta_{1}, \eta_{2}$ is the effective viscosity of the layer and half-space respectively.

2.2. The stresses satisfy the following equations: (assuming body forces do not change during our investigation)

$$
\begin{aligned}
& \frac{\partial}{\partial y_{2}}\left(\tau_{22}^{k}\right)+\frac{\partial}{\partial y_{3}}\left(\tau_{23}^{k}\right)=0, \\
& \frac{\partial}{\partial y_{2}}\left(\tau_{32}^{k}\right)+\frac{\partial}{\partial y_{3}}\left(\tau_{33}^{k}\right)=0
\end{aligned}
$$

where, for the layer $\left(-\infty<y_{2}<\infty, 0 \leq y_{3} \leq H, t \geq 0\right)$.

And for the half-space, $\left(-\infty<y_{2}<\infty, y_{3} \geq H, t \geq 0\right)$ and assuming quasi-static deformation for which the inertia terms are neglected.

2.3. The boundary conditions are taken as, with $t=0$ representing an instant when the medium is in aseismic state

$$
\begin{aligned}
& \tau_{22}^{k}\left(y_{2}, y_{3}, t\right)=\tau_{\infty}(t) \cos \theta \quad \text { for } \quad-\infty<\mathrm{y}_{2}<\infty, \quad t \geq 0, \\
& \text { and } \quad 0 \leq y_{3} \leq H \quad \text { for } \quad k=1, \quad y_{3} \geq H \quad \text { for } \quad k=2 .
\end{aligned}
$$

On the free surface $y_{3}=0,\left(-\infty<y_{2}<\infty, t \geq 0\right)$

$$
\begin{aligned}
& \tau_{23}^{l}\left(y_{2}, y_{3}, t\right)=0, \\
& \tau_{33}^{l}\left(y_{2}, y_{3}, t\right)=0 .
\end{aligned}
$$

Also as $y_{3} \quad y_{3} \rightarrow \infty\left(-\infty<y_{2}<\infty, t \geq 0\right)$

$$
\begin{aligned}
& \tau_{23}^{2}\left(y_{2}, y_{3}, t\right)=0, \\
& \tau_{33}^{2}\left(y_{2}, y_{3}, t\right)=\tau_{\infty}(t) \sin \theta
\end{aligned}
$$


where, $\quad k=1,2$

$$
\tau_{33}^{1}\left(y_{2}, y_{3}, t\right)=\tau_{33}^{2}\left(y_{2}, y_{3}, t\right)=\tau_{H} \text { (say) } \quad \text { at } \quad y_{3}=H,\left(-\infty<y_{2}<\infty, t \geq 0\right),
$$

and

$$
w_{1}\left(y_{2}, y_{3}, t\right)=w_{2}\left(y_{2}, y_{3}, t\right) \quad \text { at } \quad y_{3}=H,\left(-\infty<y_{2}<\infty, t \geq 0\right)
$$

[where $\tau_{\infty}(t)$ is the shear stress maintained by mantle convection and other tectonic phenomena throughout the medium].

\subsection{The initial conditions are}

Let $\left((v)_{1}\right)_{0},\left((w)_{1}\right)_{0},\left((v)_{2}\right)_{0},\left((w)_{2}\right)_{0},\left(\tau_{r s}^{2}\right)_{0} \quad$ and $\quad\left(e_{r s}^{2}\right)_{0} r, s=2,3 \quad$ be the value of $\left((v)_{1}\right),\left((w)_{1}\right),\left(v_{2}\right)$ and $\left(w_{2}\right),\left(\tau_{r s}^{2}\right)$ and $\left(e_{r s}^{2}\right)$ respectively at time $t=0$ which are functions of $y_{2}, y_{3}$ and satisfy the relations $(2.1)-(2.12)$.

\subsubsection{Solutions in the absence of any fault dislocation}

The boundary value problem given by Eqs (2.1)-(2.12), can be solved by taking the Laplace transformation with respect to time ' $t$ ' of all the constitutive equations and the boundary conditions. On taking the inverse Laplace transformation we get the solutions for displacements, stresses as :

For $M 1$

$$
\begin{aligned}
& v_{1}\left(y_{2}, y_{3}, t\right)=\left(v_{1}\right)_{0}+\left[\left(y_{3}\right) / \mu_{1}\right] \times\left[\tau_{\infty}(t)-\tau_{\infty}(0)\right] \times \cos \theta, \\
& w_{1}\left(y_{2}, y_{3}, t\right)=\left(w_{1}\right)_{0}+\left(y_{3} / \mu_{1}\right) \times\left[\tau_{\infty}(t)-\tau_{\infty}(0)\right] \times \sin \theta, \\
& \tau_{22}^{l}\left(y_{2}, y_{3}, t\right)=\tau_{\infty}(t) \cos \theta, \quad \tau_{23}^{l}\left(y_{2}, y_{3}, t\right)=0, \\
& \tau_{33}^{l}\left(y_{2,} y_{3}, t\right)=\tau_{\infty}(t) \sin \theta .
\end{aligned}
$$

For M2: (Sen and Debnath, 2012)

$$
\begin{aligned}
& v_{2}\left(y_{2}, y_{3}, t\right)=\left(v_{2}\right)_{0}+y_{2}\left(\cos \theta / \mu_{2}\right)\left[\left(\tau_{\infty}(t)-\tau_{\infty}(0)\right)+\left(\mu_{2} / \eta_{2}\right) \int_{0}^{t} \tau_{\infty}(\tau) d \tau\right] \\
& w_{2}\left(y_{2} y_{3}, t\right)=\left(w_{2}\right)_{0}+\left(y_{3} / \mu_{2}\right)\left[\left(\tau_{\infty}(t)-\tau_{\infty}(0)\right)+\left(\mu_{2} / \eta_{2}\right) \int_{0}^{t} \tau_{\infty}(\tau) d \tau\right] \sin \theta, \\
& \tau_{22}^{2}=\tau_{\infty}(t) \cos \theta-\left[\tau_{\infty}(0) \cos \theta-\left(\tau_{22}^{2}\right)_{0}\right] e^{-\left(\mu_{2} / \eta_{2}\right) t}, \\
& \tau_{23}^{2}=\left(\tau_{23}^{2}\right)_{0} e^{-\left(\mu_{2} / \eta_{2}\right) t}, \quad \tau_{33}^{2}=\left[\tau_{\infty}(t)-\tau_{\infty}(0) e^{-\left(\mu_{2} / \eta_{2}\right) t}\right] \sin \theta .
\end{aligned}
$$


From the above solution we find that $\tau_{22}^{2}$ increases with time and tends to $\tau_{\infty}(t) \cos \theta$ as $t$ tends to $\infty$, while $\tau_{23}^{2}$ tends to zero, but $\tau_{33}^{2}$ tends to $\tau_{\infty}(t) \sin \theta$. We assume that the geological conditions as well as the characteristic of the fault is such that when $\tau_{2^{\prime} 3^{\prime}}$ reaches some critical value, say $\tau_{c}<\tau_{\infty}(t) \cos \theta$ the fault $F$ undergoes a sudden slip along the dip direction.

The magnitude of the sudden slip shall satisfy the following conditions as discussed by Mukhopadhyay et al. (1980a).

(C1) Its value will be maximum on the free surface.

(C2) The magnitude of the slip will decrease with $y_{3}$ as we move downwards and ultimately tends to zero near the lower edge of the fault.

$$
\left(y_{2}^{\prime}=0, y_{3}^{\prime}=D\right) \text {. }
$$

\subsubsection{Solutions after the fault movements: (Debnath and Sen, 2013)}

We assume that after a time $T_{1}$, the stress component $\tau_{2^{\prime} 3^{\prime}}^{2}$ (which is the main driving force for the dip-slip motion of the fault) exceeds the critical value $\tau_{c}$, and the fault $F$ undergoes a sudden slip along the dip direction, characterized by a dislocation across the fault given in Appendix-A.

We solve the resulting boundary value problem by modified Green's function method following Maruyama (1966), Rybicki $(1971 ; 1973)$ and correspondence principle (as shown in the Appendix-A) and get the solution for displacements, stresses and strain as:

For M1

or,

$$
\begin{aligned}
& v_{l}\left(y_{2}, y_{3}, t\right)=\left(v_{1}\right)_{0}+\left[\left(y_{3}\right) / \mu_{1}\right] \times\left[\tau_{\infty}(t)-\tau_{\infty}(0)\right] \times \cos \theta, \\
& w_{l}\left(y_{2}, y_{3}, t\right)=\left(w_{1}\right)_{0}+\left(y_{3} / \mu_{1}\right) \times\left[\tau_{\infty}(t)-\tau_{\infty}(0)\right] \times \sin \theta+ \\
& +(U /(2 \times \pi)) \times \int_{F} g\left(x_{3}^{\prime}\right)\left[\left(A_{1} / B_{1}\right)+\left(C_{1} / D_{1}\right)\right]-\sum_{l}^{\infty}\left(\frac{\bar{\mu}_{1}-\bar{\mu}_{2}}{\bar{\mu}_{1}+\bar{\mu}_{2}}\right)^{m} \times \varphi\left(y_{2}^{\prime}, y_{3}^{\prime}, \theta\right) d x_{3}^{\prime}, \\
& \left.w_{l}\left(y_{2}, y_{3}, t\right)=\left(w_{1}\right)_{0}+\left(y_{3} / \mu_{1}\right) \times\left[\tau_{\infty}(t)\right)-\tau_{\infty}(t)\right] \times \sin \theta+\psi \text { (say) }
\end{aligned}
$$

where,

$$
\begin{aligned}
& \bar{\psi}=\text { Laplace transformation of } \psi \\
& =(U /(2 \times \pi)) \times \int_{F} g\left(x_{3}^{\prime}\right)\left[\left(A_{1} / B_{1}\right)+\left(C_{1} / D_{1}\right)\right]-\sum_{1}^{\infty}\left(\frac{\bar{\mu}_{1}-\bar{\mu}_{2}}{\bar{\mu}_{1}+\bar{\mu}_{2}}\right)^{m} \varphi\left(y_{2}^{\prime}, y_{3}^{\prime}, \theta\right) d x_{3}^{\prime} .
\end{aligned}
$$

Therefore taking the inverse Laplace transformation we get, 


$$
\begin{aligned}
& \psi=(U /(2 \times \pi)) \times \int_{F} g\left(x_{3}^{\prime}\right) \times\left[\left(A_{1} / B_{1}\right)+\left(C_{1} / D_{1}\right)\right]+ \\
& -\sum_{1}^{\infty}(a / b)^{m}\left[1+\sum_{r=1}^{m}\left(\begin{array}{l}
m \\
r
\end{array}\right) \frac{b_{1}^{r}}{(r-1) !} t^{r-1} e^{-a_{1} t}\right]\left[A_{--}+A_{-+}+A_{+-}+A_{++}\right]
\end{aligned}
$$

and

$$
\begin{array}{ll}
A_{1}=\left(y_{2}\right) \sin \theta-\left(y_{3}\right) \cos \theta, & B_{1}=\left[\left(x_{3}^{\prime}\right)^{2}-2\left(x^{\prime}\right)_{3}\left(y_{2} \cos \theta+y_{3} \sin \theta\right)+\left(y_{2}^{2}\right)+\left(y_{3}^{2}\right)\right], \\
C_{1}=\left(y_{2}\right) \sin \theta+\left(y_{3}\right) \cos \theta, & D_{1}=\left(\left(x_{3}^{\prime}\right)^{2}\right)-2\left(x_{3}^{\prime}\right)\left(y_{2} \cos \theta-y_{3} \sin \theta\right)+\left(y_{2}^{2}\right)+\left(y_{3}^{2}\right)
\end{array}
$$

where

$$
\begin{aligned}
& \varphi\left(y_{2}^{\prime}, y_{3}^{\prime}, \theta\right)=\left(\left(A_{1}\right)_{-+} /\left(B_{1}\right)_{-+}\right)+\left(\left(A_{1}\right)_{--} /\left(B_{1}\right)_{--}\right)+ \\
& -\left(\left(C_{1}\right)_{+-} /\left(\left(D_{1}\right)\right)_{+-}\right)-\left(\left(C_{1}\right)_{++}\right) /\left(\left(D_{1}\right)_{++}\right)=A_{-+}+A_{--}+A_{+-}+A_{++} \text {(say), }
\end{aligned}
$$

and

$$
\begin{aligned}
& A_{-+}=\left(\left(A_{1}\right)_{-+} /\left(B_{1}\right)_{-+}\right), \quad A_{--}=\left(\left(A_{1}\right)_{--} /\left(\left(B_{1}\right)_{--}\right),\right. \\
& A_{+-}=-\left(\left(C_{1}\right)_{+-} /\left(\left(D_{1}\right)_{+-}\right), \quad A_{++}=-\left(\left(C_{1}\right)_{++}\right) /\left(\left(D_{1}\right)_{++}\right),\right.
\end{aligned}
$$

$\left(A_{1}\right)_{-+}=\left(y_{2}\right) \sin \theta-\left(2 \times m \times H+y_{3}\right) \cos \theta$,

$\left(B_{1}\right)_{-+}=\left[\left(x_{3}^{\prime 2}\right)-2\left(x_{3}^{\prime}\right)^{2}\left(y_{2} \cos \theta+\left(2 \times m \times H+y_{3}\right) \sin \theta\right)+\left(y_{2}^{2}+\left(2 \times m \times H+y_{3}\right)^{2}\right)\right]$,

$\left(A_{1}\right)_{--}=\left(y_{2}\right) \sin \theta-\left(2 \times m \times H-y_{3}\right) \cos \theta$,

$\left(B_{1}\right)_{--}=\left[\left(x_{3}^{\prime 2}\right)-2\left(x_{3}^{\prime}\right)^{2}\left(y_{2} \cos \theta+\left(2 \times m \times H-y_{3}\right) \sin \theta\right)+\left(y_{2}^{2}+\left(2 \times m \times H-y_{3}\right)^{2}\right)\right]$,

$\left(C_{1}\right)_{+-}=\left(y_{2}\right) \sin \theta+\left(2 \times m \times H-y_{3}\right) \cos \theta$,

$\left(D_{1}\right)_{+-}=\left(\left(x_{3}^{\prime}\right)^{2}-2\left(x^{\prime}\right)_{3}\left(y_{2} \cos \theta-\left(2 \times m \times H-y_{3}\right) \sin \theta\right)+\left(y_{2}^{2}\right)+\left(2 \times m \times H-y_{3}\right)^{2}\right)$, 


$$
\begin{aligned}
& \left(C_{1}\right)_{++}=\left(y_{2}\right) \sin \theta+\left(2 \times m \times H+y_{3}\right) \cos \theta, \\
& \left(D_{1}\right)_{++}=\left(\left(x_{3}^{\prime}\right)^{2}-2\left(x_{3}^{\prime}\right)\left(y_{2} \cos \theta-\left(2 \times m \times H+y_{3}\right) \sin \theta\right)+\left(y_{2}^{2}\right)+\left(2 \times m \times H+y_{3}\right)^{2}\right), \\
& a=\frac{\mu_{1}}{\mu_{2}}-1, \quad b=\frac{\mu_{1}}{\mu_{2}}+1, \quad a_{1}=\frac{\mu_{1}}{b} \times\left[\frac{1}{\mu_{1}}+\frac{1}{\mu_{2}}\right], \quad b_{1}=\frac{\mu_{1} \mu_{2}}{\mu_{1}^{2}-\mu_{2}^{2}} \times\left(\frac{\mu_{2}}{\eta_{2}}-\frac{\mu_{1}}{\eta_{1}}\right), \\
& a_{3}=a_{1}-\frac{\mu_{1}}{\eta_{1}}, \quad T_{r}(t)=e^{-\left(\mu_{1} / \eta_{2}\right) t} \times\left[1-e^{a_{3} t} e_{r-1}\left(a_{3} t\right)\right]
\end{aligned}
$$

where

$$
e_{r-1}\left(a_{3} t\right)=1+\left(a_{3} t\right)+\left(a_{3} t\right)^{2} / 2 !+\ldots \ldots \ldots \ldots+\left(a_{3} t\right)^{r-1} /(r-1) !
$$

and

$$
r-1 \geq 0, \quad e_{0}\left(a_{3} t\right)=1,
$$

$$
\tau_{22}^{l}\left(y_{2}, y_{3}, \theta, t\right)=\tau_{\infty}(t) \cos \theta
$$$$
\tau_{23}^{l}\left(y_{2}, y_{3}, \theta, t\right)=\mu_{1} \times \frac{\partial}{\partial y_{2}}(\psi)=\mu_{1} \times\left(\psi_{2}\right) \text { (say) }
$$

where $\quad\left(\psi_{2}\right)=\frac{\partial}{\partial y_{2}}(\psi)$

$$
\tau_{33}^{l}\left(y_{2}, y_{3}, \theta, t\right)=\tau_{\infty}(t) \sin \theta+\mu_{1} \times\left(\frac{\partial \psi}{\partial y_{2}}\right)=\tau_{\infty}(t) \sin \theta+\mu_{1} \times \psi_{3} \text { (say) }
$$

where $\quad\left(\psi_{3}\right)=\frac{\partial}{\partial y_{2}}(\psi)$

\section{Numerical computations}

Following Aki and Richards (1980) and recent studies on rheological behavior of crust and upper mantle by Chift et al. (2002), Shun-ichiro Karato (2010) the values of the model parameters are taken as:

$\mu_{1}=3 \times 10^{10}$ pascal,$\mu_{2}=3.5 \times 10^{10}$ pascal,$\eta_{1}=2 \times 10^{20}$ poise, $\eta_{2}=3 \times 10^{20}$ poise $D=$ depth of the fault $=10 \mathrm{~km}$ [noting that the depth of all major earthquake faults is between $10-15 \mathrm{~km}$ ]. $H=$ thickness of the layer $=40 \mathrm{~km}$, say (though the thickness varies from region to region of the Earth) $t_{l}=t-T_{1}$. $\tau_{\infty}(t)=2 \times 10^{7}$ pascal (200 bars), [post seismic observations reveal that stresses released in major earthquake are of the order of 200 bars, in extreme cases it may be 400 bars].

$$
\left(\tau_{23}^{1}\right)_{0}=5 \times 10^{6} \text { pascal }(50 \text { bars }) \quad \text { and } \quad \tau_{\infty}(0)=0 .
$$

We take the function $g\left(x_{3}^{\prime}\right)=W\left[\left(\left(x_{3}^{\prime}\right)^{2}-D^{2}\right)^{2}\right] /(D)^{4}$, 
with $W=1 \mathrm{~cm} /$ year, satisfying the conditions stated in $\left(C_{1}\right)-\left(C_{2}\right)$.

We now compute the following quantities:

For layer M1

$$
\begin{aligned}
& W_{1}\left(y_{2}, y_{3}, \theta, t\right)=w_{1}\left(y_{2}, y_{3}, \theta, t\right)-\left(w_{1}\right)_{0}+\left(y_{3} / \mu_{1}\right) \times\left[\tau_{\infty}(t)-\tau_{\infty}(0)\right] \times \sin \theta, \\
& t_{23}^{1}\left(y_{2}, y_{3}, \theta, t\right)=\tau_{23}^{l}\left(y_{2}, y_{3}, \theta, t\right)
\end{aligned}
$$

where, $\tau_{23}^{i}, e_{23}^{i}, e_{33}^{i}$ are given by Eq.(2.14), for $i=1$.

\section{Results and discussions}

\subsection{Variation of shear stress $t_{23}$ with depth due to the movement across $F$}

Figures (2)-(7) show the special as well as temporal variation of shear stress $t_{23}$ for various $\theta$.

\section{Spacial variation}

Equation (2.14) gives the expressions for shear stress $t_{23}$ due to the fault movement. Figure 2 shows the variation of shear stress $t_{23}$ with depth $y_{3}$ for $y_{2}=8 \mathrm{~km}, t_{1}$ (i.e., $t-T$ ) $=1$ year and $\theta=60$ (in deg.) due to the fault movement. It is observed that the shear stress $t_{23}$ gets accumulated up to a depth of about $20 \mathrm{~km}$, then releases with a very slow rate attaining a maximum with magnitude of 0.38 bar /year at a depth of about 5 $\mathrm{km}$ and then starts releasing .

Figure 3 shows the variation of shear stress $t_{23}$ with depth $y_{3}$ for $y_{2}=8 \mathrm{~km}, t_{1}$ (i.e., $t-T$ ) $=1$ year and $\theta=$ 45 (in deg.) due to the fault movement. It is observed that the shear stress gets released up to a depth of about $10 \mathrm{~km}$, then starts accumulating at a comparatively slower rate attaining a maximum at a depth of about 13 $\mathrm{km}$ with magnitude of $0.25 \mathrm{bar} /$ year and then gradually decreases to zero.

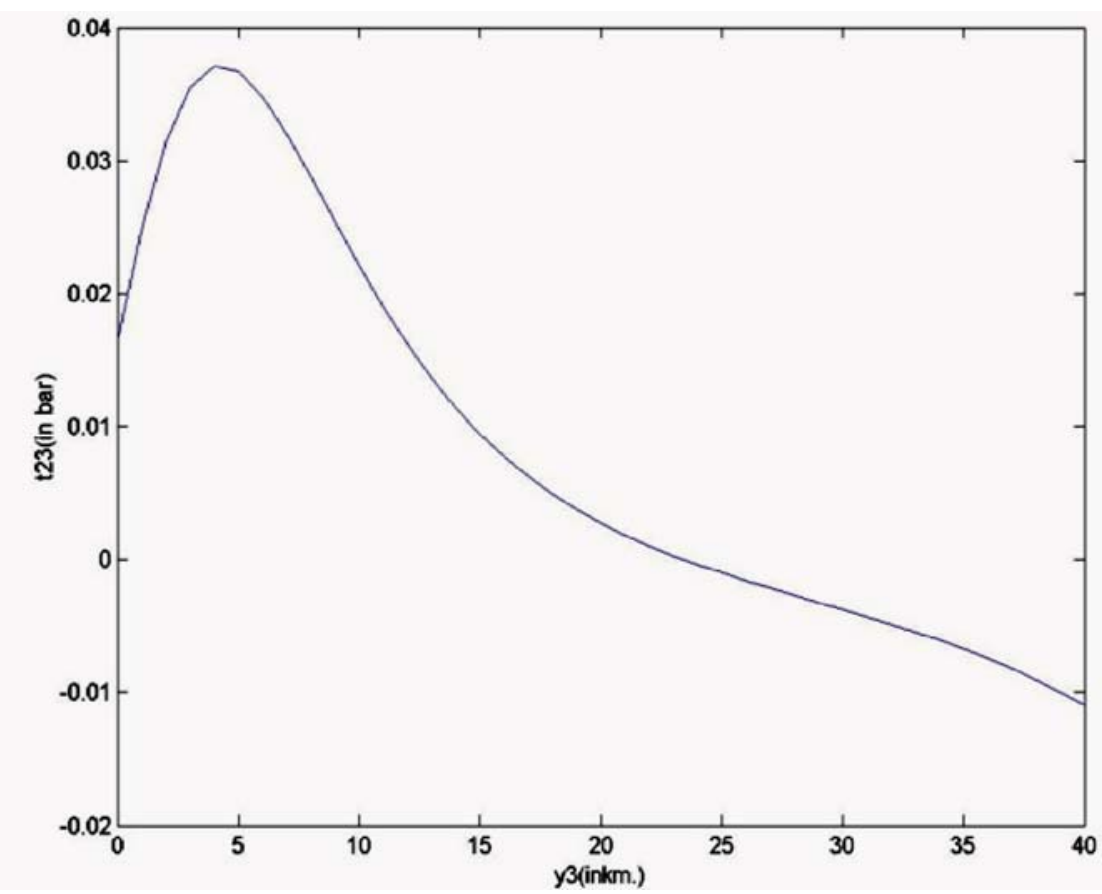

Fig.2. Variation of stress $t_{23}$ with depth $y_{3}$ for $y_{2}=8 \mathrm{~km}$. $\theta=60(\mathrm{deg}) ; t_{1}$ (i.e., $\left.t-T\right)=1$ year due to fault movement. 


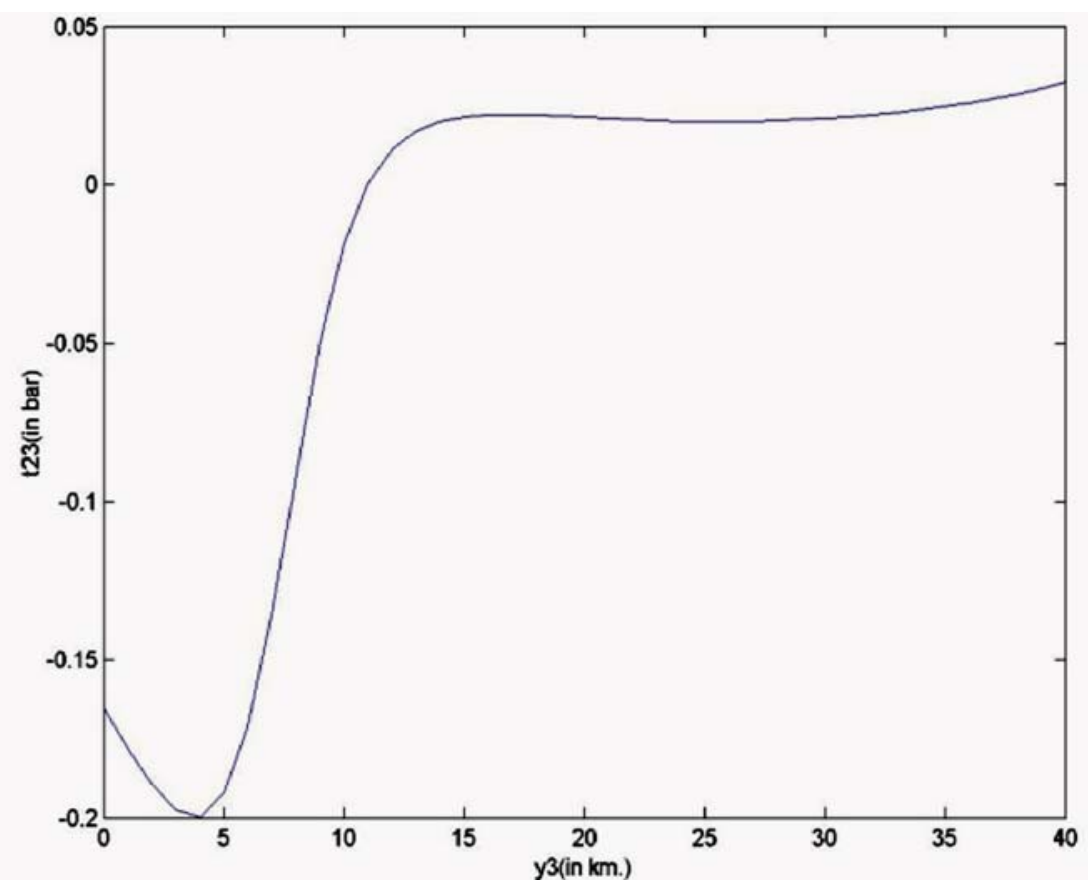

Fig.3. Variation of stress $t_{23}$ with depth $y_{3}$ for $y_{2}=8 \mathrm{~km}$. $\theta=45(\mathrm{deg}) ; t_{1}$ (i.e., $\left.t-T\right)=1$ year due to fault movement.

Figure 4 shows the variation of shear stress $t_{23}$ with depth $y_{3}$ for $y_{2}=8 \mathrm{~km}, t_{1}$ (i.e., $t-T$ ) $=1$ year and $\theta=$ 90 (in deg.), $H=40 \mathrm{~km}$ due to the fault movement. It is found that the shear stress gets released up to a depth of about $7 \mathrm{~km}$ then starts accumulating at a comparatively slower rate with a maximum accumulation of magnitude $0.02 \mathrm{bar} /$ year at a depth of about $13 \mathrm{~km}$ from the free surface.

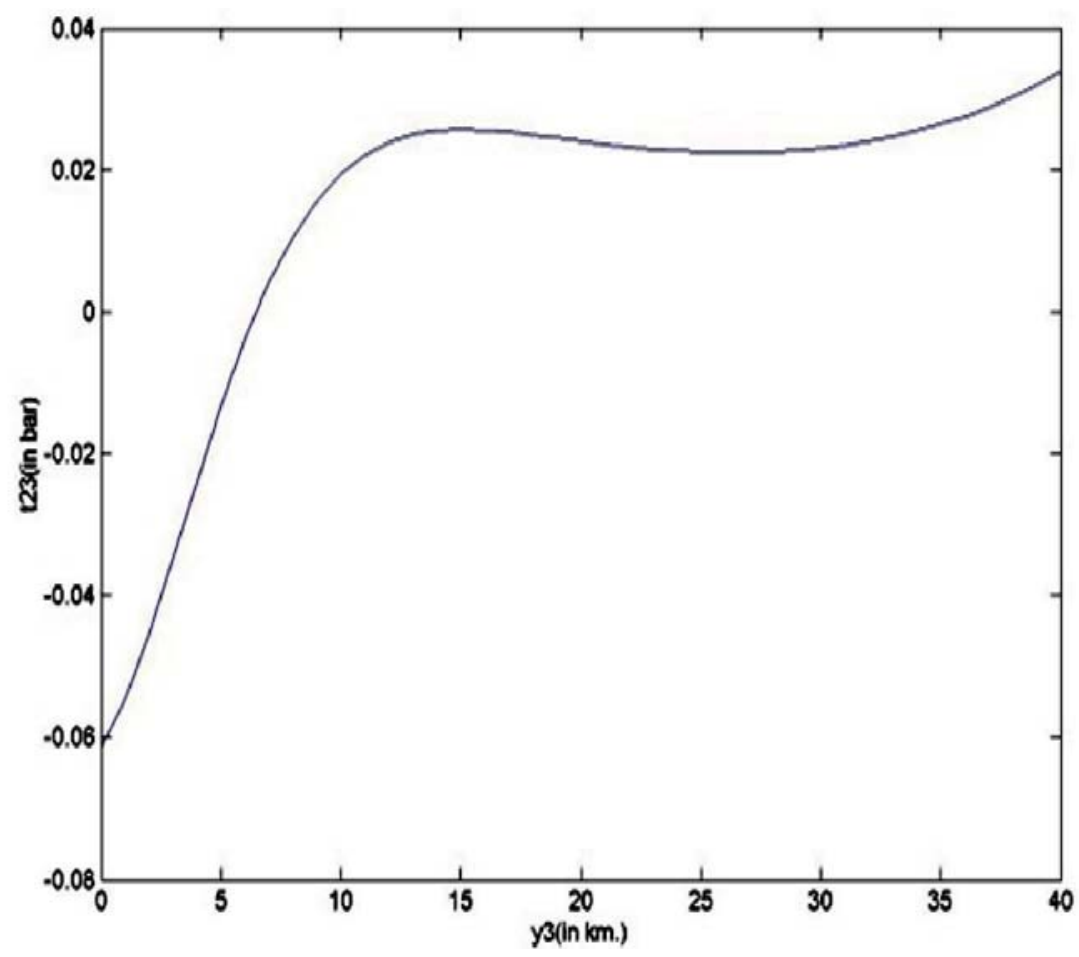

Fig.4. Variation of stress $t_{23}$ with depth $y_{3}$ for $y_{2}=8 \mathrm{~km} . \theta=90(\mathrm{deg}) ; t_{1}$ (i.e., $\left.t-T\right)=1$ year $H=40 \mathrm{~km}$ due to fault movement. 


\section{Temporal variation}

Figure 5 shows the rate of shear stress accumulation/release in the model, for $y_{3}=1 \mathrm{~km}$, for $y_{2}=8$ $\mathrm{km}, \theta=45$ (in deg.). It is observed that the rate of shear stress release increases linearly with a non vanishing initial value which is quite expected for a viscoelastic model as it possesses a past history. But Fig.6 shows that the rate of stress accumulation decreases linearly with time " $t$ " at a point where $y_{3}=1 \mathrm{~km}$ : for $y_{2}=8 \mathrm{~km}$ : $\theta=45$ (in deg.).

Figure 6 shows the rate of shear stress accumulation/release in the model, for $y_{3}=1 \mathrm{~km}$, for $y_{2}=8$ $\mathrm{km}, \theta=60$ (in deg.). It is observed that the rate of shear stress accumulation decreases linearly with a non vanishing initial value which is quite expected for a viscoelastic model as it possesses a past history. Figure 7 shows that the rate of stress release increases linearly with time " $t$ " at a point where $y_{3}=1 \mathrm{~km}$; for $y_{2}=8 \mathrm{~km}: \theta=90$ (in deg.), it is found that the pattern is the same as that of Fig.5 with a different numerical value.

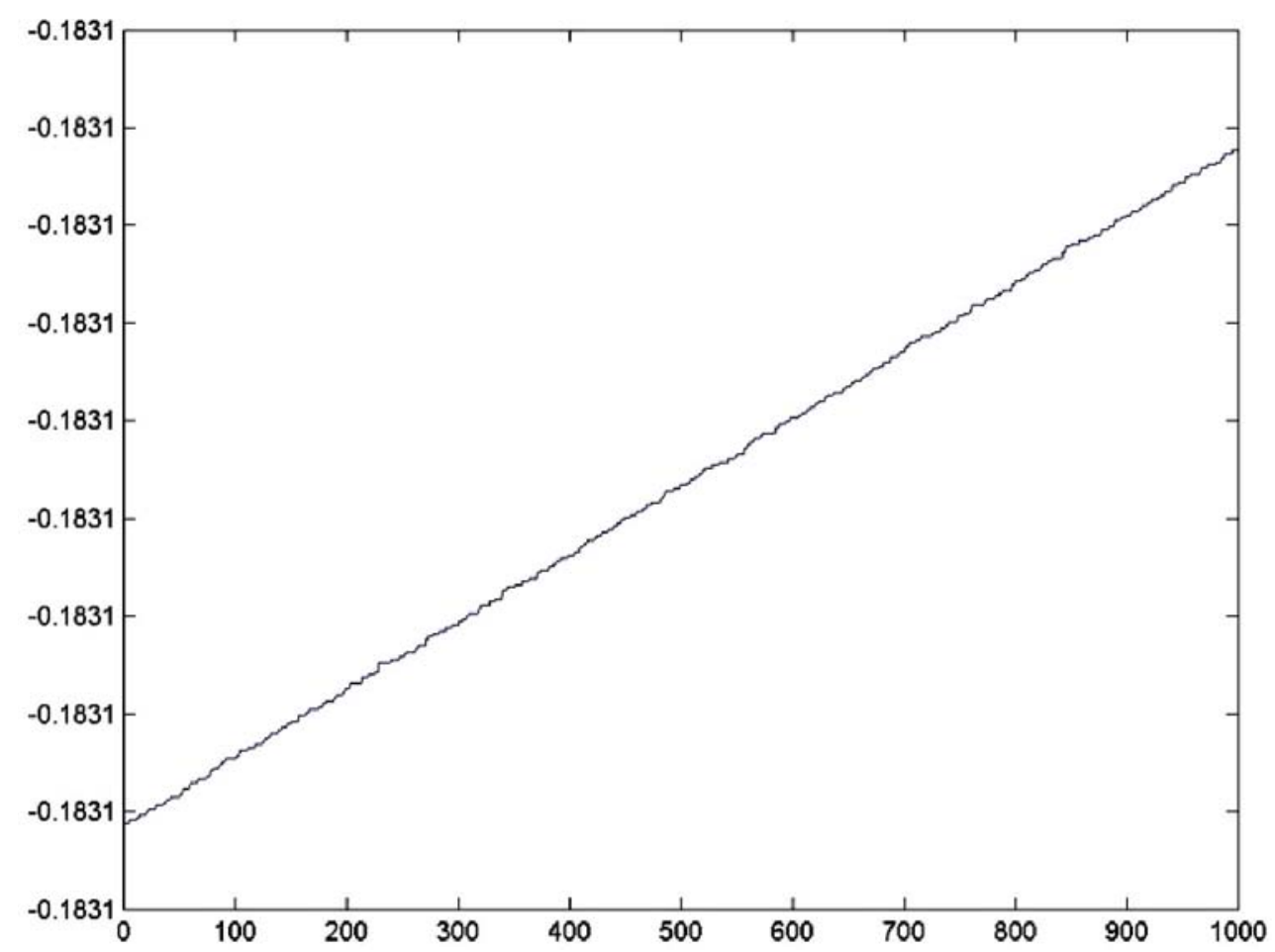

Fig.5. Rate of stress $t_{23}$ accumulation $/$ release for $y_{3}=1 \mathrm{~km}$. for $y_{2}=8 \mathrm{~km}$. $\theta=45$ (deg), due to fault movement. 


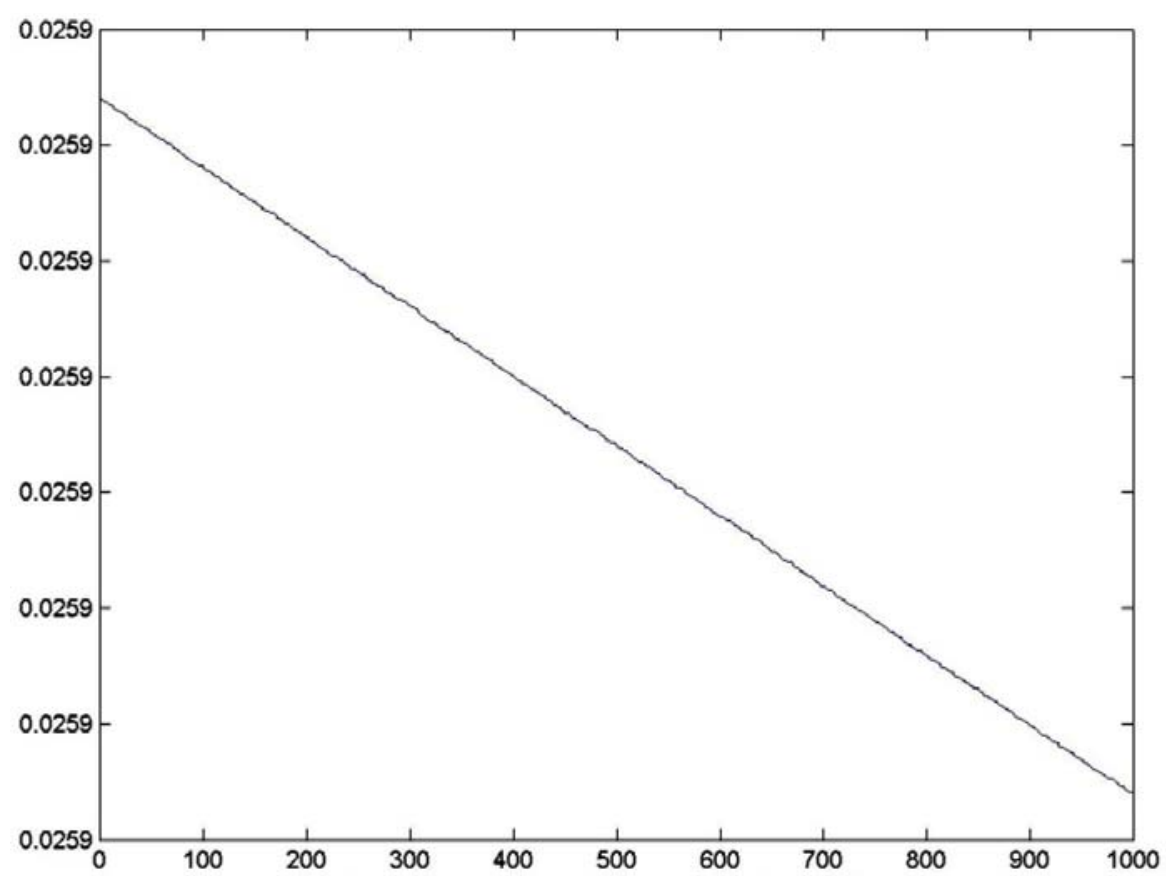

Fig.6. Rate of stress $t_{23}$ accumulation $/$ release for $y_{3}=1 \mathrm{~km}$. for $Y_{2}=8 \mathrm{~km} . \theta=60(\mathrm{deg})$, due to fault movement.

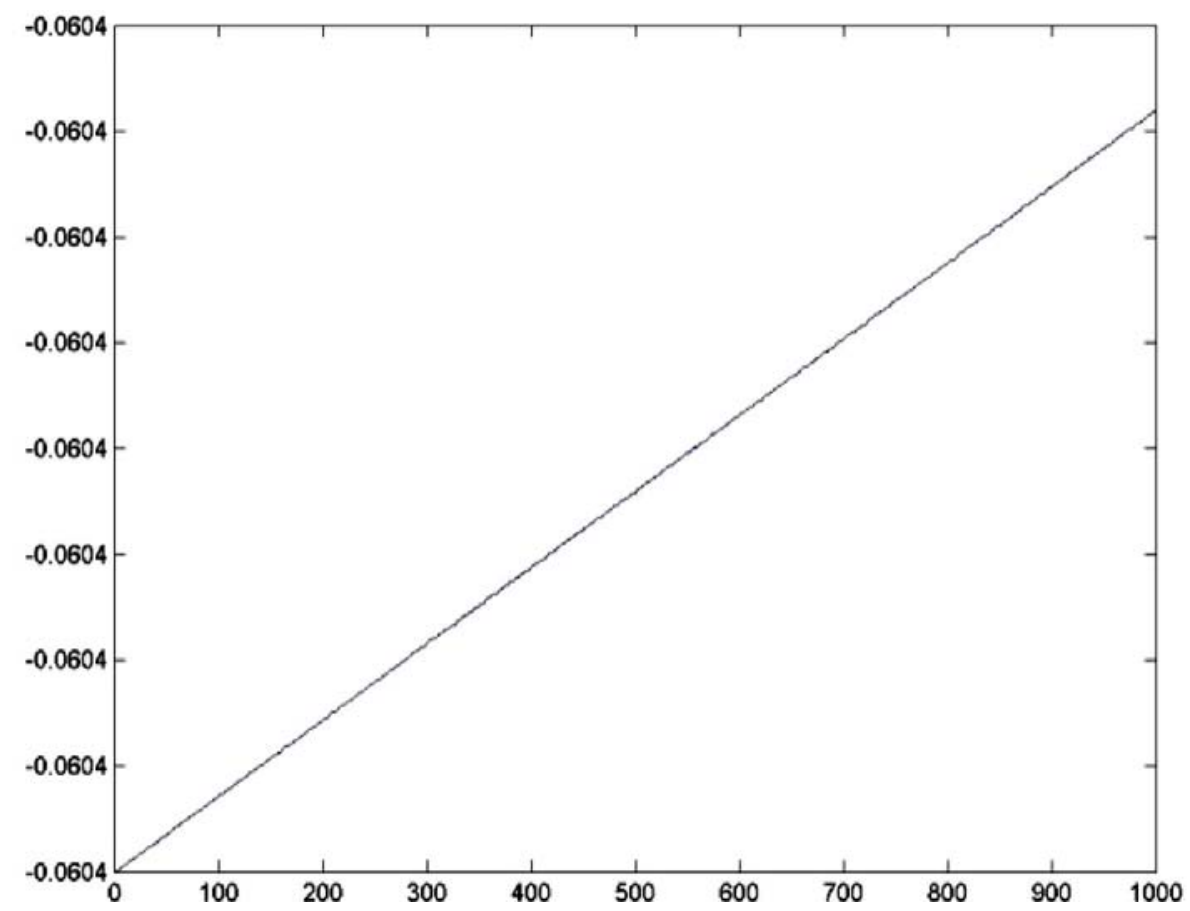

Fig.7. Rate of stress $t_{23}$ accumulation $/$ release for $y_{3}=1 \mathrm{~km}$. for $y_{2}=8 \mathrm{~km}$. $\theta=90(\mathrm{deg})$, due to fault movement.

\subsection{Effect of layer thickness on accumulation / release of shear stress $t_{23}$}

As discussed above in Fig.4 we have seen that the shear stress first gets released up to a depth of about $7 \mathrm{~km}$ then starts accumulating at a comparatively slower rate up to a depth of about $13 \mathrm{~km}$ from the free surface for $H=40 \mathrm{~km}$ but Fig. 8 shows variation of shear stress $t_{23}$ with depth $y_{3}$ for $y_{2}=8 \mathrm{~km}, t_{1}$ (i.e., $t-T$ ) $=$ 
1 year and $\theta=90$ (in deg.), $H=100 \mathrm{~km}$ due to the fault movement. We observe that the shear stress first gets released up to a depth of about $8 \mathrm{~km}$, then starts accumulating at a comparatively slower rate up to a depth of about $29 \mathrm{~km}$ attaining a maximum with magnitude $0.02 \mathrm{bar} / \mathrm{year}$ and then gradually diminishes.

Thus we conclude that the viscoelastic thickness has a major role in the accumulation/release pattern in the viscoelastic layered model and accumulation/release of stress in the region also depends on the dip angle and the position of the points with respect to the fault.

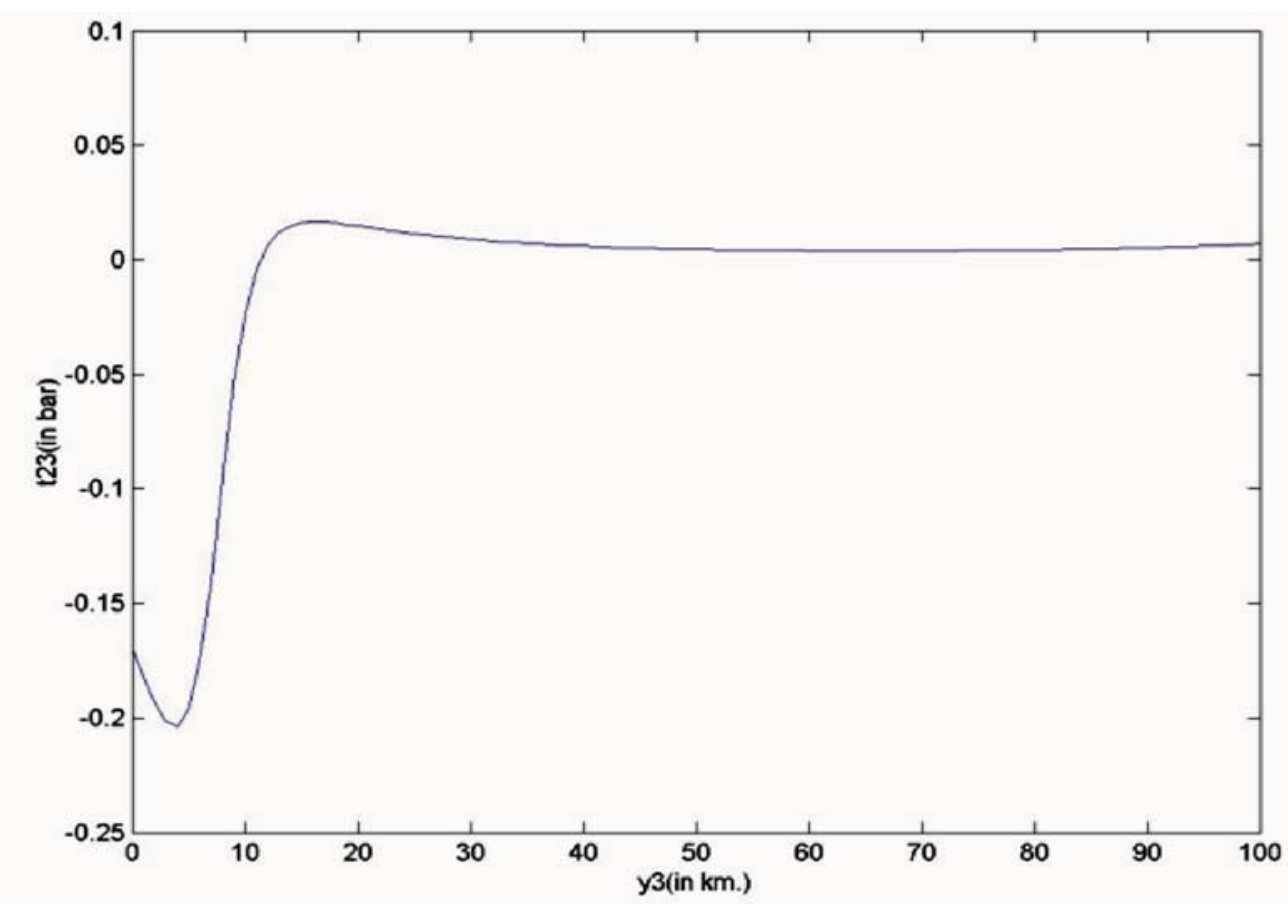

Fig.8. Variation of stress $t_{23}$ with depth $y_{3}$ for $y_{2}=8 \mathrm{~km} \theta=90(\mathrm{deg}) ; t_{1}$ (i.e., $\left.t-T\right)=1$ year, $H$ (thickness of the layer) $=100 \mathrm{~km}$ due to fault movement.

\section{Appendix-A}

\subsection{Solutions after the fault movement}

We assume that after a time $T_{1}$ the stress component $\tau_{2^{\prime} 3^{\prime}}$ (which is the main driving force for the dip-slip motion of the fault) exceeds the critical value $\tau_{c}$, the fault $F$ undergoes a sudden slip. Then we have an additional condition characterizing the dislocation in $w$ due to the slipping movement as

$$
[(w)]_{i F}=U g\left(x_{3}^{\prime}\right) H\left(t_{1}\right)
$$

where, $U$ is the maximum dislocation along the dip of the fault occurred at the free surface, it is value gradually diminishes with increasing direction of $x_{3}^{\prime}$ governed by the function $g\left(x_{3}^{\prime}\right)$ and $H\left(t_{1}\right)$ is the Heaviside function and $[(w)]_{i F}=$ The discontinuity of $w_{i}$ across $F$ is given by

$$
[(w)]_{i F}=\lim _{\left(y_{2}^{\prime} \rightarrow 0+\right)}(w)_{i}-\lim _{\left(y_{2}^{\prime} \rightarrow 0+\right)}(w)_{i}
$$


for $\quad\left(y_{2}^{\prime}=0,0 \leq y_{3}^{\prime} \leq D\right)$.

Taking the Laplace transformation in Eq.(A1) we get,

$$
\overline{[(w)]_{i F}}=\frac{U}{p} g\left(y_{3}^{\prime}\right)
$$

The fault slip commences across $F$ after time $T_{l}$, therefore,

$$
[(w)]_{i F}=0,
$$

for $t_{1} \geq 0$, where $t_{1}=t-T_{1}, F$ is located in the region $\left(y^{\prime}{ }_{2}=0,0 \leq y_{3}^{\prime} \leq D\right)$.

We try to find the solution as

$$
\begin{array}{ll}
(v)_{t}=\left((v)_{i}\right)_{1}+\left((v)_{i}\right)_{2}, & (w)_{i}=\left((w)_{i}\right)_{1}+\left((w)_{i}\right)_{2}, \\
\tau_{22}^{i}=\left(\tau_{22}^{i}\right)_{1}+\left(\tau_{22}^{i}\right)_{2}, & \tau_{23}^{i}=\left(\tau_{23}^{i}\right)_{1}+\left(\tau_{23}^{i}\right)_{2}, \quad \tau_{33}^{i}=\left(\tau_{33}^{i}\right)_{1}+\left(\tau_{33}^{i}\right)_{2}
\end{array}
$$

where $i=1,2$ and $\left((v)_{1}\right)_{1},\left((w)_{1}\right)_{1},\left(\tau_{r s}^{i}\right)_{1}$, are continuous everywhere in the model and are given by (A). While the second part $\left((v)_{i}\right)_{2}$ and $\left(\tau_{r s}^{i}\right)_{2}$ are obtained by solving the modified boundary value problem as stated below. We note that $\left((v)_{2}\right)_{2}$ is continuous even after the fault slip, so that $\left([(v)]_{2}\right)_{2}=0$, while $\left((w)_{2}\right)_{2}$ satisfies the dislocation condition given by Eq.(A2).

The resulting boundary value problem can now be stated as: $\left((v)_{1}\right)_{2}\left((w)_{1}\right)_{2}\left((v)_{2}\right)_{2}\left((w)_{2}\right)_{2}$ satisfies $2 \mathrm{D}$.

Laplace equations as

$$
\begin{array}{ll}
\nabla^{2} \overline{\left((v)_{1}\right)_{2}}=0, & \nabla^{2} \overline{\left((w)_{1}\right)_{2}}=0, \\
\nabla^{2} \overline{\left((w)_{2}\right)_{2}}=0, & \nabla^{2} \overline{\left((v)_{2}\right)_{2}}=0
\end{array}
$$

where, $r, s,=2,3$

where, $\overline{\left((w)_{2}\right)_{2}}$ is the Laplace transformation of $\left((w)_{2}\right)_{2}$, with the modified boundary conditions, For M1

$$
\begin{array}{lcrl}
\overline{\tau_{22}^{l}}\left(y_{2}, y_{3}, \theta\right)=0 & \text { as } & \left|y_{2}\right| \rightarrow \infty, & 0 \leq y_{3} \leq H, \\
\overline{\tau_{23}^{l}}\left(y_{2}, y_{3}, \theta\right)=0 & \text { as } & y_{2} \rightarrow \infty\left(-\infty<y_{2}<\infty\right), \\
\overline{\tau_{33}^{l}}\left(y_{2}, y_{3}, \theta\right)=0 & \text { as } & y_{3} \rightarrow \infty\left(-\infty<y_{2}<\infty\right),
\end{array}
$$


and the other boundary conditions are as previous.

For M1

$$
\left((w)_{1}\right)_{2}(Q)=\int_{F} U \times g\left(x_{3}^{\prime}\right)\left[G\left(y_{2}, y_{3}, x_{2}, x_{3}\right)\right] d x_{3}^{\prime}
$$

where

$$
\left[G\left(y_{2}, y_{3}, x_{2}, x_{3}\right)\right]=\frac{\partial}{\partial x_{2}} G_{1}\left(y_{2}, y_{3}, x_{2}, x_{3}\right)
$$

and

$$
G_{1}\left(y_{2}, y_{3}, x_{2}, x_{3}\right)=\left(A_{1} / B_{1}\right)+\left(C_{1} / D_{1}\right)-\sum_{1}^{\infty}\left(\frac{\bar{\mu}_{1}-\bar{\mu}_{2}}{\bar{\mu}_{1}+\bar{\mu}_{2}}\right)^{m} \times \varphi\left(y_{2}^{\prime}, y_{3}^{\prime}, \theta\right) d x_{3}^{\prime},
$$

for

$$
0 \leq y_{3} \leq H
$$

and

$$
\begin{aligned}
& A_{1}=\left(y_{2}\right) \sin \theta-\left(y_{3}\right) \cos \theta, \quad B_{1}=\left[\left(x_{3}^{\prime 2}\right)-2\left(x^{\prime}\right)_{3}\left(y_{2} \cos \theta+y_{3} \cos \theta\right)+\left(y_{2}^{2}\right)+\left(y_{3}^{2}\right)\right], \\
& C_{1}=\left(y_{2}\right) \sin \theta+\left(y_{3}\right) \cos \theta, \quad D_{1}=\left(\left(x^{\prime}\right)_{3}^{2}\right)-2\left(x_{3}^{\prime}\right)\left(y_{2} \cos \theta-y_{3} \sin \theta\right)+\left(y_{2}^{2}\right)+\left(y_{3}^{2}\right), \\
& \varphi\left(\left(y_{2}^{\prime}, y_{3}^{\prime}, \theta\right)=\left(\left(A_{1}\right)_{-+} /\left(B_{1}\right)_{-+}+\left(\left(A_{1}\right)_{--} /\left(\left(B_{1}\right)_{--}\right)-\left(\left(C_{1}\right)_{+-} /\left(D_{1}\right)_{+-}\right)-\left(\left(C_{1}\right)_{++}\right) /\left(\left(D_{1}\right)_{++}\right),\right.\right.\right. \\
& \left(A_{1}\right)_{-+}=\left(y_{2}\right) \sin \theta-\left(2 \times m \times H+y_{3}\right) \cos \theta, \\
& \left(B_{1}\right)_{-+}=\left[\left(x_{3}^{\prime 2}\right)-2\left(x_{3}^{\prime}\right)^{2}\left(y_{2} \cos \theta+\left(2 \times m \times H+y_{3}\right) \sin \theta\right)+\left(y_{2}^{2}+\left(2 \times m \times H+y_{3}\right)^{2}\right)\right]
\end{aligned}
$$

where,

$$
\begin{aligned}
& \left(A_{1}\right)_{--}=\left(y_{2}\right) \sin \theta-\left(2 \times m \times H-y_{3}\right) \cos \theta, \\
& \left(B_{1}\right)_{--}=\left[\left(x_{3}^{\prime 2}\right)-2\left(x_{3}^{\prime}\right)^{2}\left(y_{2} \cos \theta+\left(2 \times m \times H-y_{3}\right) \sin \theta\right)+\left(y_{2}^{2}+\left(2 \times m \times H-y_{3}\right)^{2}\right)\right], \\
& \left(C_{1}\right)_{+-}=\left(y_{2}\right) \sin \theta+\left(2 \times m \times H-y_{3}\right) \cos \theta, \\
& \left(D_{1}\right)_{+-}=\left(\left(x_{3}^{\prime}\right)^{2}-2\left(x_{3}^{\prime}\right)\left(y_{2} \cos \theta-\left(2 \times m \times H-y_{3}\right) \sin \theta\right)+\left(y_{2}^{2}\right)+\left(2 \times m \times H-y_{3}\right)^{2}\right), \\
& \left(C_{1}\right)_{++}=\left(y_{2}\right) \sin \theta+\left(2 \times m \times H+y_{3}\right) \cos \theta, \\
& \left(D_{1}\right)_{++}=\left(\left(x_{3}^{\prime}\right)^{2}-2\left(x_{3}^{\prime}\right)\left(y_{2} \cos \theta-\left(2 \times m \times H+y_{3}\right) \sin \theta\right)+\left(y_{2}^{2}\right)+\left(2 \times m \times H+y_{3}\right)^{2}\right),
\end{aligned}
$$




$$
\begin{aligned}
& \left.w_{1}\left(y_{2}, y_{3}, t\right)=\left(w_{1}\right)_{0}+\left(y_{3} / \mu_{1}\right) \times\left[\tau_{\infty}(t)\right)-\tau_{\infty}(t)\right] \times \sin \theta+ \\
& +(U /(2 \times \pi)) \times \int_{F} g\left(x_{3}^{\prime}\right) \times\left[\left(A_{1} / B_{1}\right)+\left(C_{1} / D_{1}\right)+\right. \\
& -\sum_{1}^{\infty}(a / b)^{m}\left[1+\sum_{r=1}^{m}\left(\begin{array}{l}
m \\
r
\end{array}\right) \frac{b_{1}^{r}}{(r-1) !} t^{r-1} e^{-a_{1} t}\right]\left[A_{--}+A_{-+}+A_{+-}+A_{++}\right] .
\end{aligned}
$$

Similarly the stress components can be found out, which are given in Eq.(2.14).

\section{Acknowledgement}

One of the authors (Subrata Kr. Debnath) thanks the Principal and Head of the Department of Basic Science and Humanities, Meghnad Saha Institute of Technology, a unit of Techno India Group (INDIA), for allowing him to pursue the research, and also thanks the Geological Survey of India, and ISI, Kolkata, for providing library facilities, the computer centre, Department of Applied Mathematics, University of Calcutta for providing computational facilities.

\section{Nomenclature}

$D$ - depth of the fault

$H$ - thickness of the layer (taken in $\mathrm{km}$ )

$M_{1}$ - layer

$M_{2}$ - half-space

$t_{23}$ - part of $\tau_{23}$ due to the fault movement only

$U$ - dislocation which is a constant in our paper

$v_{k}$ and $w_{k}$ - displacement component along the horizontal and vertical axes for $k=1$ for the layer and $k=2$ for the half-space respectively

$\theta$ - dip angle.(taken in degree)

$\mu_{k}, \eta_{k}$ - effective rigidity and viscosity for $k=1$ for the layer and $k=2$ for the half-space respectively

$\tau^{\prime}, \tau$ - stress in the 'prime' and 'unprimed 'system respectively

$\tau_{2^{\prime} 3^{\prime}} \quad$ - stress component in 'prime' system

$\tau_{c}-$ critical value of the stress i.e., the value of the stress after which fault movement occur

$\tau_{i j}^{k}, e_{i j}^{k}, i, j=2,3-$ stress and strain tensor for $k=1$ for the layer and $k=2$ for the half-space respectively

$\tau_{\infty}(t)$ - tectonic force which arises due to mantle convection or any other tectonic phenomenon

\section{References}

Aki K. and Richards P.G. (1980): Quantitative seismology. theory and methods. - W.H. Freeman, San Francisco, California.

Cathles L.M. (1975): The visco-elasticity of the Earth's mantle. - Princeton, N. J.: Princeton University Press.

Chift P., Lin J. and Barcktiausen U. (2002): Marine and petroleum geology.- Vol.19, pp.951-970.

Cohen (1980a): Post seismic viscoelastic surface deformations and stress,1. - Theoretical Considerations, Displacements and Strains Calculations, J. Geophys.Res.85, No. B6, 3131-3150.

Debnath S.K and Sen S. (2013): Two interacting creeping vertical rectangular strike-slip faults in a viscoelastic half space model of the lithosphere. - IJSER, vol.4, No.6, p.(Accepted).

Debnath S.K. (2013): A buried vertical rectangular finite fault in a viscoelastic layer over a viscoelastic half-space and its influence on stress-strain accumulation. - JEES (under review). 
Debnath S.K. and Sen S. (2013): Aseismic ground deformation in a viscoelastic layer overlying a viscoelastic halfspace model of the lithosphere. - Asthenosphere System Geosciences, vol.3(2), pp.60-67.

Debnath S.K. and Sen S. (2013): Stress and strain accumulation due to a long dip-slip fault movement in an elastic layer over a viscoelastic half-space model of the lithosphere-asthenosphere system. - International Journal of Geosciences, vol.4, No.3, p.(Accepted).

Fairbridge (1961): Eustatic changes in sea-level. - Physics and Chemistry of the Earth, pp.99-185, Pergamon Press, London(edited by L.N. Ahrens, F. Press, K. Rankama and S.K. Runcorn).

Fuis S.G., Scheirer S.D., Langenheim E.V. and Kohler D.M. (2012): A new perspective on the geometry of the San Andreas fault of South California and relationship to lithospheric structure. - B.S.S.A vol.102, pp.1236-1251.

Ghosh U. and Sen S. (2011): Stress accumulation near buried fault in lithosphere-asthenosphere system. - International Journal of Computing, vol.1, No.4, pp.786-795.

Ghosh U., Mukhopadhyay A. and Sen S. (1992): On two interacting creeping vertical surface-breaking strike-slip faults in a two-layered model of lithosphere. - Physics of the Earth and Planetary Interior, vol.70, pp.119-129.

Maruyama T. (1966): On two dimensional dislocations in an infinite and semi- infinite medium. - Bull. Earthquake Res. Inst. Tokyo Univ., vol.44, (part 3), pp.811-871.

Maruyama T. (1964): Statical elastic dislocations in an infinite and semi-infinite medium. - Bull. Earthquake. Res. Inst., Tokyo Univ., vol.42, pp.289-368.

Mukhopadhyay A. et al. (1979a): On stress accumulation near finite rectangular fault. - Indian Journal of Meteorology, Hydrology and Geophysics (Mausam), vol.30, pp.347-352.

Mukhopadhyay A. et al. (1979b): On stress accumulation and fault slip in lithosphere. - Indian Journal of Meteorology, Hydrology and Geophysics(Mausam), vol.30, pp.353-358.

Mukhopadhyay A., Sen S. and Paul B.P. (1980,a): On stress accumulation in a viscoelastic lithosphere containing a continuously slipping fault. - Bull. Soc. Earthquake Technology, vol.17, No.1, pp.1-10.

Mukhopadhyay A., Sen S. and Paul B.P. (1980a): On stress accumulation near a continuously slipping fault in a two layered model of lithosphere. - Bull. Soc. Earthquake Technology, vol.17, No.4, pp.29-38.

Nur A. and Mavko G. (1974): Post-seismic viscoelastic rebound. - Science, vol.183, pp.204-206.

Oglesby D.D. (2005): The dynamics of strike-slip step-overs with linking dip-slip faults. - B.S.S.A, vol.95, No.5, pp.1604-1622.

Rani S. and Singh S.J. (1992): Static deformation of a uniform half space due to a long dip-slip fault Geophy. - J. Int., vol.109, pp.469-476.

Rani S., Singh S.J. and Garg N.R. (1991): Displacements and stress at any point of uniform half space due to two dimensional buried source. - Phys. Earth. Planet. Int., vol.65, pp.276-282.

Rundle J.B. (1978): Viscoelastic crustal deformation by finite quasi-static sources. - Journal of the Geophysical Research, vol.83, No.B12, pp.5937-5947.

Rybicki K. (1971): The elastic residual field of a very long strike slip fault in the presence of a discontinuity. - Bull. Seis. Soc. Am. vol.61, pp.79-92.

Sato R. (1972): Stress drop of finite fault. - J. Phys. Earth, vol.20, pp.397-407.

Sato R. and Yamashita T. (1975): Static deformations in an obliquely layered medium Part-II dip-slip fault - Journal of the Phys. of the Earth, vol.23, pp.113-125.

Savage J.C. (1998): Displacement field for an edge dislocation in layered half space. - Journal of Geophysical Research, vol.103, No.B2, pp.2439-2446.

Savage J.C. (2007): Post-seismic relaxation associated with transient creep Rheology. - J. Geophys. Res., B5, vol.112, B05412.

Savage J.C. and Hastie L.M. (1966): Surface deformation associated with dip slip faulting. - J.G.R71, No.20, pp.48974904. 
Schofield J.C. (1964): Post-glacial sea level and iso-static uplift. - New Zealand: J. Geol. Geopgysics, vol.7, pp.359370.

Segal P. (2010): Earthquake and Volcano Deformation. - Princeton: University Press.

Sen S. and Debnath S.K. (2012): A creeping vertical strike- slip fault of finite length in a viscoelastic half-space model of the lithosphere. - International Journal of Computing, vol.2, No.3, pp.687-697.

Sen S. and Debnath S.K. (2012): Long dip-slip fault in a viscoelastic half-space model of the lithosphere. - American Journal of Computational and Applied Mathematics, vol.2, No.6, pp.249-256.

Sen S., Sarker S. and Mukhopadhyay A. (1993): A creeping and surface breaking long strike-slip fault inclined to the vertical in a viscoelastic half space. - Mausam, vol.44,4, pp.365-4,372.

Shun-Ichiro K. (2010): Rheology of the Earth's mantle. - A historical review Gondwana Research-vol.18-Issue-1, pp.17-45.

Shuo M.A., Gregory C. and Beroza (2008): Rupture dynamics on a bimaterial interface for dipping faults. - Bulletin of the Seismological Society of America, vol.98, pp.1642-1658.

Singh S.J. and Rosenman M. (1974): Quasi static deformation of a viscoelastic half -space by a displacement dislocation. - Phys. of the Earth and Planetary Interiors, vol.8, pp.87-101.

Singh S.J., Punia M. and Kumari G. (1997): Deformation of a layered half-space due to a very long dip-slip fault. Proc. Indian Natn. Sci. Acad., vol.63A, No.3, pp.225-240.

Tomar S.K. and Dhiman (2003): 2D-deformation analysis of a half-space due to a very long dip-slip fault at finite depth. - Indian Acad. Sci.(Earth. Planet, Sci.) vol.112, No.4, pp.587-596.

Zhang C., Oglesby D.D. and XU G. (2006): Earthquake nucleation on dip-slip faults with depth-dependent frictional properties. - Journal of Geophysical Research, vol.111, No.10, B07303.

Received: May 16, 2013

Revised: June 12, 2016 